\title{
MUDANÇA DOS CRITÉRIOS QUALIS!
}

Editorial. Mudança dos critérios Qualis! Arq Gastroenterol. 2010;47(2):125-7.

DESCRITORES - Publicações periódicas como assunto. QUALIS CAPES.

A Associação Médica Brasileira (AMB) preocupada com o futuro das publicações científicas brasileiras, depois da divulgação dos novos critérios QUALIS da CAPES, vem, desde agosto de 2009, organizando uma série de encontros em sua sede em São Paulo, em parceria com a Associação Brasileira de Editores Científicos - ABEC Brasil. Os encontros resultaram em um editorial denominado Classificação dos periódicos no sistema QUALIS da CAPES - a mudança dos critérios é URGENTE! Este foi assinado por 62 editores de revistas científicas e publicado na íntegra em todas elas e em outras inúmeras principalmente na área da saúde, evidenciando uma sensibilização e um envolvimento cada vez maior dos periódicos nacionais em discutir problemas comuns ${ }^{(1)}$.

A comunidade científica continua preocupada com as perspectivas, os rumos e o futuro dos periódicos brasileiros ${ }^{(2,3)}$. Assim, na reunião de 18 de março de 2010 os editores presentes puderam avaliar as repercussões do primeiro Editorial que serviu de base para discussões em eventos e reuniões científicas pelo país. Esta última reunião contou com a participação da Dra. Lilian Caló, Coordenadora de Comunicação Científica e Avaliação do SciELO, que apresentou estudo comparativo dos periódicos brasileiros na referida base, classificados por dois critérios: o primeiro conforme o fator de impacto ISI/ JCR, que usa somente as revistas indexadas na base Thomson Reuters, e segundo um índice composto pela somatória simples dos fatores de impacto ISI/ JCR e do SciELO. O fator de impacto SciELO, que também considera citações de todos os periódicos da sua base, modifica significativamente o número de citações obtidas e, consequentemente, eleva o fator de impacto dos periódicos brasileiros. Este fato ficou mais evidente com a demonstração apresentada pela Dra. Caló do ganho percentual obtido pelos periódicos com a adoção do índice composto. Fica claro que associar outros índices, criar equivalências ou alternativas diversas podem favorecer a qualificação das revistas nacionais, melhorando sua visibilidade e favorecendo a indexação internacional. Também deve se considerar que os pesquisadores nacionais estão preferindo publicar seus conteúdos em revistas estrangeiras ao invés de fazê-los em revistas nacionais.
Esta escolha, melhora a qualificação do Programa de Pós-graduação aos quais estão inseridos, conquistam fator de impacto mais elevado e aumentam o índice $\mathrm{H}$; tudo isso única e exclusivamente por conta dos novos critérios adotados pela CAPES. A busca de maior visibilidade e qualidade da produção nacional não deve ser avaliada somente pelos artigos, mas também por maior qualificação de nossos periódicos para que eles sejam reconhecidos internacionalmente.

Considerando que para atual avaliação trienal da CAPES os critérios já estão definidos, os editores reunidos decidiram elaborar novo editorial contendo uma lista de sugestões a ser encaminhada para a coordenação da CAPES para a próxima avaliação. A lista de sugestões que complementam a do primeiro editorial é a seguinte:

- revisão dos critérios usados pela CAPES para classificação dos periódicos, sugerindo que seja adotado o fator de impacto composto pela somatória dos fatores de impacto ISIIJCR e SciELO;

- obtenção de um assento para a ABEC Brasil (Associação Brasileira dos Editores Científicos) no Conselho Técnico Cientifico da CAPES, para que os editores possam ser ouvidos no processo;

- solicitação da "Bolsa do Editor" junto ao CNPq para auxílio à editoração científica destinada a editores de revistas brasileiras que recebem apoio da referida agência de fomento. Este recurso tem por objetivo aprimorar a qualidade das revistas obtendo maior dedicação de seus editores às funções editoriais;

Além disto, os editores reunidos decidiram obter apoio da Academia Brasileira de Ciências, da FINEP e do Deputado Eleuses Vieira de Paiva para suas reivindicações e sugestões. Num segundo momento os editores solicitarão ao $\mathrm{CNPq}$ detalhamento dos resultados e dos critérios adotados para distribuição dos recursos dos Editais para Auxílio à Editoração (AED). Com estas informações os editores pretendem construir um banco de dados com informações sobre orçamentos anuais dos periódicos brasileiros que será útil para análise comparativa e cooperação mútua. A divulgação dos dois editoriais e sua discussão continua sendo nossa meta em buscar o reconhecimento que os periódicos nacionais necessitam e merecem. 
Editorial. Change the Qualis criteria! Arq Gastroenterol. 2010;47(2):125-7.

HEADINGS - Periodicals as topic. QUALIS CAPES.

\section{REFERÊNCIAS}

1. Classificação dos periódicos no sistema QUALIS da CAPES - A mudança dos critérios é urgente! Arq Gastroenterol. 2010;47:4-6.
2. de Lucena AF, Tibúrcio RV. Qualis periódicos: visão do acadêmico na graduação médica. Rev Assoc Med Bras. 2009;55: 247-8.

3. Rocha-e-Silva M. O novo Qualis, ou a tragédia anunciada. Clinics. 2009;64: $1-4$.
Assinam este editorial:

Adagmar Andriolo Jornal Brasileiro de Patologia e Medicina Laboratorial

Alfredo José Afonso Barbosa Jornal Brasileiro de Patologia e Medicina Laboratorial

Antonio Carlos lopes Revista da Sociedade Brasileira de Clínica Médica

Arnaldo José Hernandez Revista Brasileira de Medicina do Esporte

Aroldo F. Camargos Revista Femina

Benedito Barraviera Journal of Venomous Animals and Toxins including Tropical Diseases

Bogdana Victoria Kadunc Surgical \& Cosmetic Dermatology da Soc. Brasileira de Dermatologia

Bruno Caramelli Revista da Associação Médica Brasileira

Carlos Brites Brazilian Journal of Infectious Diseases

Dejair Caitano do Nascimento Hansenologia Internationalis

Domingo M. Braile Revista Brasileira de Cirurgia Cardiovascular

Dov Charles Goldenberg Revista Brasileira de Cirurgia Plástica

Edmund Chada Baracat Revista da Associação Médica Brasileira

Edson Marchiori Revista Radiologia Brasileira

Eduardo de Paula Vieira Revista Brasileira de Coloproctologia

Flávia Machado Revista Brasileira de Terapia Intensiva
Geraldo Pereira Jotz

Revista Brasileira de Cirurgia Cabeça e Pescoço Aquivos Internacionais de Otorrinolaringologia

Gianna Mastroianni Kirsztajn Jornal Brasileiro de Nefrologia

Gilberto Camanho Revista Brasileira de Ortopedia

Gustavo Gusso Medicina de Família e Comunidade

Ivomar Gomes Duarte Revista de Administração em Saúde

Izelda Maria Carvalho Costa Anais Brasileiros de Dermatologia

João Ferreira de Mello Júnior Brazilian Journal of Otorhinolaryngology

Joel Faintuch Revista Brasileira de Nutrição Clínica

José Antônio Baddini Martinez Jornal Brasileiro de Pneumologia

José Antonio Livramento Arquivos de Neuro-Psiquiatria

José Eduardo Ferreira Manso Revista do Colégio Brasileiro de Cirurgiões

José Eulálio Cabral Filho Revista Brasileira de Saúde Materno Infantil

José Heverardo da Costa Montal Revista da Associação Brasileira de Medicina de Tráfego

José Luiz Gomes do Amaral Revista da Associação Médica Brasileira

José Luiz Martins Archives of Pediatric Surgery

Jurandyr Moreira de Andrade Revista Brasileira de Ginecologia e Obstetrícia

Leonardo Cançado Monteiro Savassi Revista Brasileira de Medicina de Família e Comunidade 
Luis dos Ramos Machado Arquivos de Neuro-Psiquiatria

Luiz Augusto Casulari Brasilia Médica

Luiz Eugenio Garcez Leme Geriatria \& Gerontologia

Luiz Felipe P. Moreira Arquivos Brasileiros de Cardiologia

Luiz Henrique Gebrim Revista Brasileira de Mastologia

Marcelo Madeira Revista Brasileira de Mastologia

Marcelo Riberto Revista Acta Fisiátrica

Marcus Bastos Jornal Brasileiro de Nefrologia

Mário Cícero Falcão Revista Brasileira de Nutrição Clínica

Mario J. da Conceição Revista da Sociedade Brasileira de Anestesiologia

Mauricio Rocha e Silva Clinics

Milton Artur Ruiz Revista Brasileira de Hematologia e Hemoterapia

Milton K. Shibata Arquivos Brasileiros de Neurocirurgia

Mittermayer Barreto Santiago Revista Brasileira de Reumatologia

Nelson Adami Andreollo ABCD Arquivos Brasileiros de Cirurgia Digestiva

Nivaldo Alonso Brazilian Journal of Craniomaxilofacial Surgery

Osvaldo Malafaia ABCD Arquivos Brasileiros de Cirurgia Digestiva
Olavo Pires de Camargo Acta Ortopedica Brasileira

Paulo Manuel Pêgo Fernandes São Paulo Medical Journal

Regina Helena Garcia Martins Brazilian Journal of Otorhinolaryngology

Renato Soibelmann Procianoy Jornal de Pediatria

Ricardo César Pinto Antunes Revista da Sociedade Brasileira de Cancerologia

Ricardo Fuller Revista Brasileira de Reumatologia

Ricardo Guilherme Viebig Arquivos de Gastroenterologia

Ricardo Nitrini Dementia \& Neuropsychologia

Rogério Dedivitis Revista Brasileira de Cirurgia Cabeça e Pescoço

Ronaldo Damião Urologia Contemporânea

Rosângela Monteiro Revista Brasileira de Cirurgia Cardiovascular

Sergio Lianza Revista Medicina de Reabilitação

Sigmar de Mello Rode Brazilian Oral Research

Tarcisio E.P. Barros Filho Acta Ortopedica Brasileira

Wallace Chamon Arquivos Brasileiros de Oftalmologia

Winston Bonetti Yoshida Jornal Vascular Brasileiro

Zuher Handar Revista Brasileira de Medicina do Trabalho 